\title{
Physical simulation of space objects' spectral characteristics for solving the reverse problem of their photometry
}

\author{
Andrey K. Murtazov \\ Astronomical observatory, Ryazan State University, Ryazan, Russia
}

Email address:

a.murtazov@rsu.edu.ru

\section{To cite this article:}

Andrey K. Murtazov. Physical Simulation of Space Objects' Spectral Characteristics for Solving the Reverse Problem of their Photometry. American Journal of Modern Physics. Vol. 2, No. 6, 2013, pp. 282-286. doi: 10.11648/j.ajmp.20130206.12

\begin{abstract}
The reverse problem of the Solar system atmosphereless body and satellite photometry is the determination of their shapes, dimensions, and reflective properties based on the observed light curves. In general, the solution of the problem is not unambiguous. To disambiguate it, the author used the physical simulation method for obtaining satellite surface spectral indicatrices. This paper describes spectral characteristics of some space object surfaces which make it possible to identify them by photometric and spectral observation results.
\end{abstract}

Keywords: Solar System, Atmosphereless Bodies, Photometry Reverse Problem, Physical Simulation, Surface Optical Properties

\section{Introduction}

The basic problem of the Solar system atmosphereless body and satellite photometry includes two interrelated problems: the direct one - determining the objects' expected brightness - to organize its observations, and the reverse one - determining a celestial body's surface parameters based on the results of its optical observations. The direct problem admits an unambiguous solution in case celestial body's surface optical (photometric) characteristics are pre-defined, whereas the reverse one - does not.

Generally, the astronomic object photometry's main problem reduces to solving the integral equation

$$
\Phi\left(\lambda, \vartheta, \varphi, \vartheta_{2}, \varphi_{2}\right)=\frac{1}{r_{0}^{2}} \sum_{k} \iint_{S_{k}} B_{k}\left(\lambda, \psi^{\prime}, \vartheta^{\prime}, \varphi^{\prime}\right) \cos \vartheta^{\prime} d S_{k},
$$

where all the object components are summarized. The double integral is taken over each component's visible sunlit side with regard to the components' mutual occultations ( $\vartheta$ the angle between the normal $\vec{n}$ of the object surface $\kappa$-component element and the observer direction).

The $\kappa$-component coating material solar spectral brightness is defined as follows:

The $\kappa$-component coating material solar spectral brightness is defined as follows:

$$
B_{k}(\lambda, \psi, \vartheta, \varphi)=\int_{\lambda_{1}}^{\lambda_{2}} b_{k}\left(\lambda, \lambda^{\prime}, \psi, \vartheta, \varphi\right) E_{S}\left(\lambda^{\prime}\right) d \lambda^{\prime}
$$

Here $E_{S}\left(\lambda^{\prime}\right)-$ solar radiation spectral density, $b_{k}\left(\lambda, \lambda^{\prime}, \psi, \vartheta, \varphi\right)-\kappa$-component coating material normalized spectral brightness within $\lambda_{I}$ wavelength caused by unit intensity wave incident radiation with $\lambda_{2}$ wavelength, and $\lambda$ - the wavelength of observations (Figure 1).

The coating materials' normalized spectral brightness $b_{k}\left(\lambda_{1}, \lambda_{2}, \psi, \vartheta, \varphi\right)$ is obtained from observations.

Therefore, the cores $B_{k}(\lambda, \psi, \vartheta, \varphi)$ are considered known - the direct problem solution case.

Considerable difficulties emerge while solving a reverse astronomic object photometry problem.

Thus stated, this problem (1) does not allow for any solution. 

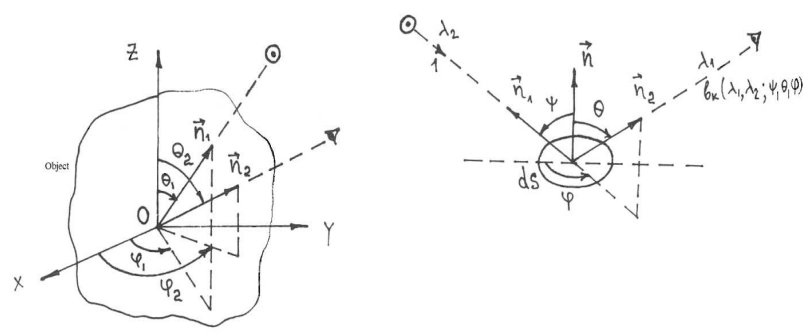

Figure 1. Vector positions in the satellite-centric reference system

It is evident that this solution should in the general case be of a probabilistic nature.

Consequently, it was solved in a simulative simplified mode considering that 1 ) the object consists of one component; 2) this component is convex; 3) this component is a body of revolution. To simplify the solution, it can be assumed that the specular reflection or Lambert diffuse scattering take place on the material surface. Thus stated, the integral equation (1) is put into the first kind standard equations of Volterra or Fredholm and is solved using standard ridge regression methods or other methods of solving incorrect problems in mathematical physics $[3,9]$.

The analysis of the equation (1) shows that it is non-linear due to: 1) the complicated dependence of the spectral brightness $B_{k}(\lambda, \psi, \vartheta, \varphi)$ on the argument; 2) the components' mutual occultations.

The unambiguous definition of a scatterer in the quantum scattering theory requires the knowledge of the scattering amplitude for all angles $\vartheta_{1}, \varphi_{1}, \vartheta_{2}, \varphi_{2}$. As any celestial body light curve $\Phi\left(\lambda, \vartheta_{1}, \varphi_{1}, \vartheta_{2}, \varphi_{2}\right)$ has the physical meaning of the electromagnetic field incoherent scattering amplitude, the unambiguous definition of the scatterer (shape, dimensions, and celestial body albedo) requires the knowledge of brightness for all of its phase angles as well as the Sun and observer orientations. To put it in other way, it is necessary to know the celestial object scattering field.

This problem is almost unsolvable in the context of practical observations. For instance, the problem for asteroids and geostationary satellites was set as follows: obtaining information about the shape, dimensions, orientation, structural element coating materials, etc. from the observed light curve (actual brightness changes versus the time function).

$$
\Phi=\Phi(\lambda, t) .
$$

Information-wise this statement of the problem is incomplete and the lack of information cannot be supplemented by internal resources. Mathematically it means that the equation (1) solution is not unique, and the problem itself is set according to Hadamart incorrectly.

\section{Physical Simulation of Space Bodies Spectral Brightness}

To disambiguate the reverse problem solution, different expert systems are created. The basic principles of their functioning amount to random comparison of photometric observation results with the results of catalogue object brightness numerical simulation.

All the conducted works related to object detection and identification with the use of the optical astronomy methods suggest the availability of an independent data bank related to the objects' surface reflection properties, which in a number of instances disambiguates the solution of their photometry's basic problem.

The author of this paper has developed a physical simulation method to obtain spectral and photometric characteristics of space object surfaces with the purpose of its further use in different expert systems of circumterrestrial space object optical monitoring $[7,11]$.

In these experiments, the space body surface sample or model is exposed to a parallel monochromatic light flux. The radiation scattered by the models was recorded by a photoelectric photometer for their different orientations and phase angles. This makes it possible to obtain a certain part of the models total scattering field, their albedo, light curves, etc.

A similar method would from time to time be applied to simulate celestial body surface light curves in order to compare the results of its application with the atmosphereless Solar system body photometry results $[6,8]$. These can also include investigations of different rocks, minerals, etc. surface optical properties [14] as applied to meteorite, asteroid, etc. surfaces.

By mid-80-ies, XX cent., a situation had been created, under which various alternatives of this method had been applied to help the solution of natural and technogenic space object photometry inverse problem. It should be noted that the first articles on physical simulation of atmosphereless celestial body and satellite photometric properties written by the author of this paper [7, 10] and NASA [1,2] were published almost simultaneously and contained similar principal requirements for experimentation and for the equipment technical parameters.

In detail the issues of solving the celestial body photometry direct problem with the use of their photometric properties simulation results were tackled upon by the author in quite a number of papers [11-13]. These papers also provide scattering indicatrices for different natural and technogenic object surfaces.

\section{Some Results}

This paper presents the results of studying satellite technogenic surfaces reflectance spectra.

Figure 2 shows some satellite coatings' spectra, resulting from our experiments: 1 - fiberglass laminate; 2, 3 glass-cloths; 4 - white titanium paint; 5 - solar cell. The values $b_{\lambda}$ are normalized relative to the Lambert magnesia coated diffuse screen. 


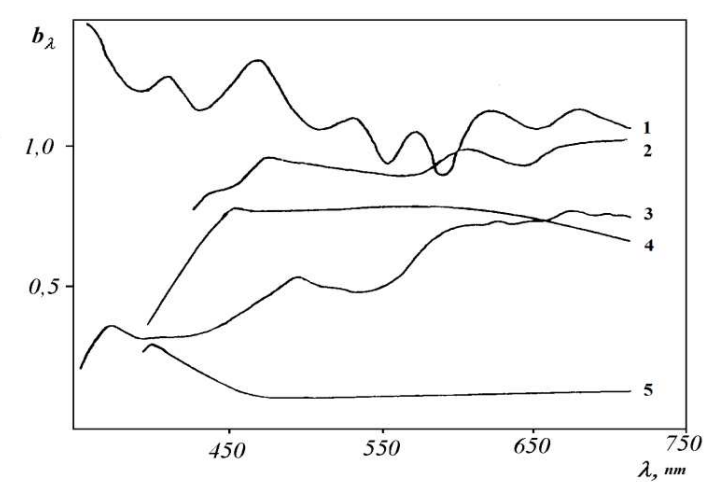

Figure 2. Some satellite reflecting coatings'spectral brightness $b_{\lambda}$

Fiberglass spectra illustrate the alternating maximums and minimums. The glass-fiber laminate's specular component makes its albedo level in some wavelengths higher than 1 . The white paint spectrum ends abruptly at wavelengths below $450 \mathrm{~nm}$. The solar panel spectrum has its strongly pronounced maximum about $420-430 \mathrm{~nm}$ - the "blue flash".

The spectral brightness $b_{\lambda}$ resulting from the experiments have been included as cores into integral equations of type (1), as well as into different expert systems for determining shapes and optical parameters of natural and technogenic body surfaces, as well as space debris surfaces (for example, [5]).

To be used in photometric observations, brightness $b_{\lambda}$-s were afterwards recalculated into colour indices.

The colour index calculation of the materials studied in the Johnson $U B V$ system was carried out as, for instance:

$$
\begin{array}{r}
a_{v}=\int_{V} B_{\lambda} d \lambda, \\
U-B=2,5 \lg \frac{\int_{B} E_{\lambda}^{0} B_{\lambda} d \lambda}{\int_{V} E_{\lambda}^{0} B_{\lambda} d \lambda},
\end{array}
$$

Here $E_{\lambda}^{0}$ и $B_{\lambda}$ are solar spectral illuminance at the distance of 1 a.u. and the normalized coating brightness $b_{\lambda}$ in corresponding system bands, $a_{V}-$ visual albedo.

Consequently, with regard to the photometric research it proved to be possible to describe the general properties of a number of satellite and technogenic space debris coatings. $[10,11]$.

Fiberglass. Phase curves are fairly complicated. A significant excess of scattered radiation is observed, especially in the direction vertical to the radiation incidence. This is apparently connected with light re-distribution in fiberglass threads. This effect is also demonstrated in spectral curves, most often in those appearing as alternate maximums and minimums which depend on the surface orientation.

Paint. The basic paint most frequently used for satellite and launch vehicle coatings is white enamel. Radiation scattering is mixed. The diffusive component is as good as the Lambert one. The amplitude of specular flashes depends on the surface coating quality and comes up to $3^{\mathrm{m}}$ with the flash width being up to $10^{\circ}$. The spectrum is rather flat with a reasonable steep slope where the wavelength is shorter than $400 \mathrm{~nm}$.

Such specifics make it possible to rather easily identify white enamels according to the results of spectral or colorimetric observations.

Glass-fiber laminate. It is very similar to fiberglass in its phase and spectral features, though has quite a remarkable specular component in its scattering.

Solar panels. The specular and diffuse components ratio is close to that of perfectly flat planes. The diffuse scattering albedo is much less than $10 \%$. The spectrum near $400 \mathrm{~nm}$ has a noticeable excess (the so-called "blue flash"). At this moment the color index $(B-V)$ becomes negative, which is an important criterion for identifying solar panels by their $B V R$-photometry. It can be noted that a visible diffuse scattering component can be found at the back side of the panels, which is important while observing the panel fragments that have become space debris.

Metals. The polished metal surface diffuse component phase characteristics are close to those of Lambert. The spectral coefficients of the metal specular reflection component are rather high: for instance, about 0.5 for steel, $0.8-0.9$ - for aluminum and titanium near $400 \mathrm{~nm}$, and 0.6 (steel), $0.8-0.9$ (aluminum and titanium) near $700 \mathrm{~nm}$.

Besides, their spectral albedo is very slightly dependent on the wavelength in visible spectrum. Hence, these materials' colour indices are close to zero and almost equal to those of the Sun.

Analysis revealed that the optical properties of satellite coating materials are quite diverse. Hereunder, with regard to different fabric data, the author managed to build up general satellite coating material classification based on their optical properties. He succeeded in distinguishing six coating classes covering quite a wide area of both albedo and colour indices $(B-V)$. The two-dimension diagram "Colour index $B-V$ - Albedo $a_{V}$ " (Figure 3 - [11]) shows the availability of quite well-marked areas occupied by certain materials: 1) white (grey) coatings; 2) black coatings (graphite/epoxy composition, fabrics); 3) yellow-green coatings; 4) red-brown coatings; 5) solar cells; 6) metal coatings.

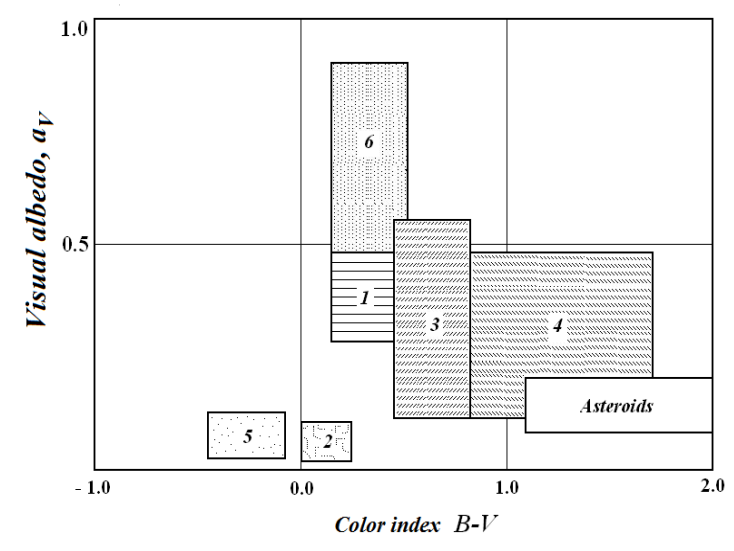

Figure 3. "Colour index $B-V$ - Albedo $a_{V}$ " for satellite surfaces 


\section{Criteria for Identifying Technogenic Debris in Circumterrestrial Space}

The availability of data on technogenic space debris' optical properties makes it possible to differentiate between technogenic and natural debris fractions (interplanetary dust, asteroid fragments, meteor matter) based on the results of multi-colour photometric observations comparative study.

Presently, the great bulk of multi-colour photometric observations is performed in the bands $B\left(\lambda_{m}=440 \mathrm{~nm}\right)$,
$V\left(\lambda_{m}=550 \mathrm{~nm}\right)$ and $R\left(\lambda_{m}=720 \mathrm{~nm}\right)$ of the Johnson photometric system.

The colour indices $B-V$ and $V-R$ determine the colour of an astronomical body's surface. In the course of the experiments in astronomical body optical properties physical simulation the author obtained the colours of satellites' basic surfaces. The surface colours of technogenic debris originating from crushed satellite surfaces should be expected the same. Table 1 of this paper shows that circumterrestrial technogenic debris colours are quite diverse - from white to red and brown.

Table 1. Optical properties of space surfaces

\begin{tabular}{|c|c|c|c|c|}
\hline $\begin{array}{l}\text { Surface } \\
\text { (type of scattering) }\end{array}$ & $\begin{array}{l}\text { Geometrical } \\
\text { albedo } \\
a_{V} \\
\end{array}$ & $\begin{array}{l}\text { Color index } \\
B-V \\
(\mathbf{m a g})\end{array}$ & $\begin{array}{l}\text { Phase curve } \\
\text { slope } \\
\text { (mag/arc deg) }\end{array}$ & Flash amplitude \\
\hline Solar cell (spec.) & 0.10 & $-0.3 \div-0.5$ & 0.013 & $10^{5}$ \\
\hline White paint (spec.) & 0.80 & $-0.05 \div 0.2$ & 0.013 & $10-30$ \\
\hline Fabric (dif.) & $0.15 \div 0.25$ & $0.2 \div 1.2$ & 0.013 & - \\
\hline Woven glass fabric (dif.) & $0.25 \div 0.75$ & $0 \div 1.2$ & $0.010 \div 0.013$ & - \\
\hline Mylar (spec.) & 0.80 & $\sim 0$ & 0.013 & $10^{2}-10^{3}$ \\
\hline Asteroids (dif.) & $0.03 \div 0.15$ & $>1.2$ & $>0.02$ & - \\
\hline
\end{tabular}

Almost all the colours of natural celestial bodies are reddish and brown-grey. Thus, the average median colour index of the Moon is $B-V=1.2$. The surfaces of most asteroids and meteoroids are even more red.

It is well known that the colour index observation data availability does not make it possible to differentiate between the surfaces of technogenic and natural debris without any additional information.

The visual topocentric magnitude $m(\varphi)$ of a circumterrestrial atmosphereless body can be presented as a linear dependence on a phase angle $\varphi$ (except those less than $5-7^{\circ}$ to exclude the opposition effect):

$$
\mathrm{m}(\varphi)=\mathrm{m}(0)+\alpha \varphi
$$

where $\alpha$ - coefficient determining the phase function slope.

The values $\alpha(\Delta m / a r c d e g)$, defined by the author for the technogenic debris surfaces are shown in Table 1. This data shows that the technogenic body surfaces light scattering in the circumterrestrial space is practically orthotropic and their phase function slopes are close to $\alpha=0,013 \mathrm{mag} / \mathrm{arc}$ deg.

Light scattering by natural atmosphereless body surfaces is far from being perfect.

In this case we deal with the effect of surface particles' mutual occultations, the tubular structure of regolith coating the surfaces, etc. Thus, phase curve slopes of atmosphereless astronomical bodies and natural debris surfaces are significantly larger than those of man-made debris and generally exceed $\alpha=0,02 \mathrm{mag} / \mathrm{arc}$ deg. [3, 4].

Earlier, the author carried out experiments which showed that bodies covered with particles of the size close to that of lunar regolith particles even with high albedo have quite large phase curve slopes, sometimes exceeding those of the Moon and asteroids [Murtazov, 1983].

The criteria of the difference between technogenic and natural surfaces in the circumterrestrial space can be stated as follows: with close colour indices $B-V, V-R$ of the observed circumterrestrial space objects the natural object surface phase function slope is at least twice as large as that of the technogenic object surface phase functions [11].

\section{Conclusion}

Using the physical simulation method the author was the first in Russia to obtain data on light scattering by technogenic debris surface coatings, the spectral and photometric characteristics of radiation scattered by them.

\section{References}

[1] V. Ambrosio, R. Burchi, et al., "Laboratory Photometry of Asteroids and Atmosphereless Bodies" Astronomy. \& Astrophysics, Vol. 44, pp. 427-430 (1985).

[2] M.A. Barucci, M. Fulchignoni, R. Salvatori, "Asteroid Photometry Simulated in the Laboratory: Phase Functions of Some Meteorites Used as Irregular Asteroid Models" Lunar and Planet. Sciences, Vol. 15, pp. 35-36 (1984).

[3] V.M. Grigorevsky, S.Ya. Kolesnik, "Light reflection from regularity specular-surface space objects" Solar System Research, Vol. 12, pp. 107-119 (1978). 
[4] B.H. Hapke, "A Theoretical Photometric Function for the Lunar Surface" Journal of Geophysical Research, Vol. 68, pp. 4571-4586 (1963).

[5] G.N. Kolesnichenko, A.V. Mironov, A.A. Vasjunin, S.V. Loginov, "Application of multispectral photometric data for recognition problem of space objects" Information-measuring and control systems, Radiotekhnika, Vol. 8, n. 5, pp. 86-92 (2010).

[6] N.I. Koshkin, "Laboratory modeling of asteroids' light curves” Solar System Research, Vol. 22, pp. 159-166 (1988).

[7] V.I. Kuryshev, A.K. Murtazov, S.I. Vereshchyagin, "Modeling of astronomical objects photometric characteristics" USSR higher school proceedings. Instrument engineering, Vol. 28, pp. 81-85 (1985).

[8] M.S. Mattews, "The Asteroid Conference in Tucson" Sky and Telescope, Vol. 42. pp. 22-24 (1971).

[9] A.P. Mosin, "Solar light regular reflection by regular-surface convex body" Astronomical Circular, n. 1459, pp. 3-5 (1986).
[10] A.K. Murtazov, "Laboratory photometry of bright diffuse cylinders" USSR higher school proceedings. Physics, Deposit manuscript, № 6178, 8 pp. (1983).

[11] A.K. Murtazov, "Optical properties of artificial space objects and technogenic space debris" Near-Earth astronomy and the problems of investigations of small bodies in the Solar system. Collected Papers, Moscow. Kosmosinform, pp. 262-268 (2000).

[12] A.K. Murtazov, "Ecology of circumterrestrial space" Moscow, Fizmatlit, 304 pp. (2004).

[13] A.K. Murtazov, "Direct photometric problem of optical monitoring of objects in circumterrestrial space" Russian Physics Journal, NY, Springer, Vol. 49, n. 11. pp. 1224-1229 (2006).

[14] P. Oetking, "Photometric Studies of Diffuse Reflecting Surfaces with Applications to the Brightness of the Moon" Journal of Geophysical Research, Vol. 71, pp. 2505-2513 (1966). 